\title{
Efectividad de un programa grupal psicoeducativo para la depresión mayor en atención primaria: ensayo clínico controlado aleatorizado.
}

\author{
Rocío Casañas, Rosa Catalán, Antonia Raya, Jordi Real \\ rocio.casanas@chmcorts.com
}

La prevalencia de la depresión mayor en Europa es del 8,56\% (1) y específicamente del 5-16\% en pacientes de atención primaria (AP), siendo la tercera causa de consulta en la AP de salud (2). La mayoría de guías de práctica clínica (GPC) para el manejo de la depresión recomiendan intervenciones psicoeducativas y psicoterapias breves como primer paso de acción en el protocolo de tratamiento (3). En relación a la psicoeducación, existe evidencia en el tratamiento de la depresión en el adulto (3-4), en la reducción de los síntomas depresivos y en la prevención de la depresión en pacientes de AP (5). Sin embargo, existen pocos estudios controlados que hayan incluido los aspectos del cuidado personal y estilo de vida saludable (dieta, ejercicio físico, dormir) conjuntamente con técnicas cognitivo-conductuales dentro de la psicoeducación y que hayan demostrado ser eficaces.

El objetivo principal de nuestro estudio (6) era evaluar la efectividad de esta intervención en pacientes con síntomas depresivos en AP y determinar si la mejora en los síntomas depresivos estaba asociada con una mejora en la calidad de vida. También estábamos interesados en analizar qué tipo de población se podría beneficiar más de esta intervención, si la población con síntomas "leves" o "moderados".

Este es un estudio multicéntrico aleatorizado, controlado, que se llevó a cabo entre Diciembre del 2008 y Abril del 2010, en 12 equipos de AP de Barcelona. Se incluyeron 231 pacientes mayores de 20 años con un diagnóstico de depresión mayor según el ICD-10 y sintomatología depresiva leve/moderada según el Beck Depression Inventory ( $\mathrm{BDI} \geq 10 \mathrm{y}<30$ ), que eran reclutados a través del médico y la enfermera de AP. Del total de pacientes, 85 tenían depresión leve (BDI $\leq 18)$ y 146 depresión moderada (BDI $\geq 19)$.

El grupo de intervención $(\mathrm{GI})(\mathrm{n}=119)$ recibió un programa grupal psicoeducativo (12 sesiones, semanales, 90 minutos, liderados por dos enfermeras) y el grupo control $(\mathrm{GC})(\mathrm{n}=112)$ recibió la atención habitual.

Los pacientes fueron evaluados al inicio del estudio y a los 3, 6 y 9 meses. Las principales medidas de resultado fueron el BDI, EuroQoL-5D y la remisión de los síntomas basado en el BDI (BDI $\leq 11)$. 
Los análisis en relación a la remisión de los síntomas mostraron diferencias significativas entre grupos, sobre todo en el grupo de síntomas leves, con una alta tasa de 57\% (p=0,009) en el post-tratamiento y del 65\% ( $\mathrm{p}=0,006)$ a los 9 meses en el GI versus $31 \%$ y $48 \%$ respectivamente en el GC. En el grupo de síntomas moderados las tasas de remisión en el GI fueron de menor magnitud con un $24 \%$ y $29 \%$ ( $\mathrm{p}=0.007$ y $\mathrm{p}=0.068$ ) respectivamente. En relación al BDI, el GI con síntomas leves mostró diferencias significativas a corto $(\mathrm{p}=0.016)$ y largo plazo $(\mathrm{p}=0.048)$ con un tamaño del efecto moderado, y el GI de síntomas moderados sólo a corto plazo.

En el EQ-5D se obtuvo una diferencia entre grupos de 5 puntos a corto y largo plazo en la muestra de pacientes leves y sólo a corto plazo los moderados, pero estas no fueron significativas.

Los resultados muestran que la intervención psicoeducativa es más eficaz en pacientes con síntomas leves, ya que presentan una mayor tasa de remisión de los síntomas a corto y largo plazo y esta mejora se asocia con una mejora en la calidad de vida. En los pacientes con síntomas moderados, la intervención no demuestra ser tan efectiva a largo plazo. Es una intervención que se recomienda en el ámbito de AP y puede ser llevada a cabo por enfermeras con una formación previa.

\section{BIBLIOGRAFÍA}

(1) Ayuso-Mateos JL, Vazquez-Barquero JL, Dowrick C, Lehtinen V, Dalgard OS, Casey P, et al: Depressive disorders in Europe: prevalence figures from the ODIN study .The British Journal of Psychiatry 2001, 179: 308-316.

(2) Rucci P, Gherardi S, Tansella M, Piccinelli M, Berardi D, Bisoffi G et al.: Subthreshold psychiatric disorders in primary care: prevalence and associated characteristics. J Affect Disord 2003, 76: $171-81$.

(3) The British Psychological Society \& The Royal College of Psychiatrists. The treatment and management of depression in adults (updated edition) National Collaborating Centre for Mental Health commissioned by the National Institute for Health \& Clinical Excellence (NICE), 2009.

(4) Cuijpers P, Andersson G, Donker T, van Straten A: Psychological treatment of depression: Results of a series of meta-analyses. Nord J Psychiatry 2011, Early Online:1-11.

(5) Cuijpers P, Muñoz RF, Clarke GN, Lewinsohn PM: Psychoeducational treatment and prevention of depression: The "Coping with Depression" course thirty years later. Clin Psychol Rev 2009, 29:449 - 58 .

(6) Casañas R, Catalán R, Val JL, Real J, Valero S, Casas M. Effectiveness of a psychoeducational group program for major depression in primary care: a randomized controlled trial. BMC Psychiatry. 2012 Dec 18;12(1):230. 